\title{
INTRODUCCIÓN A LA METODOLOGÍA DE LA INVESTIGACIÓN EN BIOÉTICA. SUGERENCIAS PARA EL DESARROLLO DE UN PROTOCOLO DE INVESTIGACIÓN CUALITATIVA INTERDISCIPLINARIA
}

\begin{abstract}
María de la Luz Casas Martínez*
Resumen: El avance de la bioética requiere de la ciencia para su desarrollo. La investigación en bioética parte de la interdisciplinariedad, lo cual complica su realización. Uno de los puntos conflictivos para aquellos que se inician en la investigación es la constitución de un adecuado protocolo. En este trabajo se pretende exponer un panorama general sobre los pasos de la integración de un protocolo con base en la investigación cualitativa.
\end{abstract}

Palabras clave: bioética, investigación, protocolo

\section{INTRODUCTION TO INVESTIGATION'S METHODOLOGY IN BIOETHICS. HINTS FOR THE DEVELOPMENT OF AN INTERDISCLINARY QUALITATIVE PROTOCOL}

Abstract: Bioethics' advance requires science's support for its development. Bioethics' investigation stems from interdisciplinarity which, of course, complicates its fulfilment. One of the items in conflict for beginners in the investigation field is the establishment of a protocol. This work pretends to state a general overview about the steps that have to be taken to integrate a protocol based on qualitative investigation.

Key words: bioethics, investigation, protocol

\section{INTRODUÇÃO À METODOLOGIA DA PESQUISA EM BIOÉTICA. SUGESTÓES PARA O DESENVOLVIMENTO DE UM PROTOCOLO DE PESQUISA QUALITATIVA INTERDISCIPLINAR}

Resumo: O desenvolvimento da ciência provoca o avanço da bioética. A pesquisa em bioética parte da interdisciplinaridade, a qual complica sua realização. Um dos pontos conflitivos para iniciantes na pesquisa é a constituição de um protocolo adequado. Este trabalho pretende expor um panorama geral sobre os passos da integração de um protocolo com base na pesquisa qualitativa.

Palavras chave: bioética, pesquisa, protocolo

Jefe del Departamento de Bioética, Escuela de Medicina, Universidad Panamericana, México

Correspondencia: mcasas@up.edu.mx 
La bioética, al igual que otras disciplinas, plantea problemas y busca respuestas. La forma de buscar estas respuestas es la metodología científica, a la cual podríamos definir como el procedimiento ordenado que se sigue para descubrir, demostrar y aportar un conocimiento científicamente válido. Científico es el conocimiento obtenido en forma sistemática, evitando la subjetividad.

Los tipos tradicionales de metodología -cuantitativa y cualitativa-, ambos con amplia validez científica, poseían cerrados campos de actuación. Sin embargo, siendo la bioética, por definición, un campo con pretensiones transdisciplinarias, debe utilizar variadas estrategias de investigación. Si bien esto puede ser enriquecedor, también complica la labor del investigador en esta disciplina.

La mayoría de las veces podemos observar que las presentaciones de bioética en los simposios o congresos llegan solamente a la interdisciplina, esto es, a presentar un tema bajo varios aspectos (médico, filosófico, jurídico, entre otros), esperando que el oyente realice análisis, síntesis y elaboración posterior de un nuevo conocimiento, el que pretendería ser transdisciplinar. Mucho se discute sobre interdisciplina, quizás porque se presenta como un intento de superar el estado actual de abstracción de las ciencias. Cuando nos referimos a interdisciplina hablamos de la cooperación de varias actividades para la búsqueda de la verdad a través del diálogo y la discusión, y en esto la bioética participa como lugar de reflexión.

La noción de multidisciplina alude a la idea de juntar saberes, donde cada uno dice lo suyo pero el producto final no es más que la exposición de todos ellos, de modo tal que cada uno conserva su individualidad. La interdisciplina, en cambio, avanza planteando la noción de funcionamiento sistémico. Esta mirada resultará de articular estas perspectivas para lograr un objeto total que exprese todas las partes. Es sabido que la simple yuxtaposición de disciplinas o su encuentro casual no es interdisciplina. Por el contrario, se requiere de una construcción conceptual común del problema y, para su operatividad, la formación de un equipo con tiempo de trabajo en común.

En el plano individual, exige renunciar a la convicción de que la propia disciplina es suficiente para dar cuenta del problema. Éste es un dato determinante, pues la interdisciplina(1) no es posible si no existe una rela- ción de los pesos que cada disciplina puede aportar. La transdisciplinariedad, finalmente, es el producto de una investigación referida a un problema definido conceptualmente de manera interdisciplinaria.

Los campos que es necesario incluir en una tesis de bioética son variados, pero siempre deben contemplar la ética, el ámbito de conocimiento específico y, de preferencia, los aspectos jurídicos, tratando de concluir en un nuevo conocimiento aplicado a los temas elegidos en el estudio. La investigación en bioética debe poseer varios ejes y todos ellos deberán aportar a la confección de una perspectiva ética. El tema puede ser tratado desde el punto de vista filosófico, médico, social, psicológico, jurídico o legal, pero al final las conclusiones deberán quedar dentro de la ética, con aplicación a los campos de conocimiento integrados en el estudio.

Desde mi experiencia como profesor del posgrado en bioética, quisiera compartir algunas observaciones que, pienso, han sido de utilidad para los alumnos que se inician en investigación o que se encuentran elaborando su protocolo. Estas recomendaciones tienen carácter simplemente de orientación. Por la extensión, no es posible desglosar siquiera en forma exhaustiva uno solo de los pasos de la metodología, tampoco abarcar todo tipo de investigación cualitativa, especialmente la filosófica. Por tanto, me referiré sólo a la que, en mi experiencia, los alumnos eligen con más frecuencia, que es la de corte sociológico, aunque sus bases pueden ser aplicadas, en general, a todo tipo de investigación cualitativa.

Como actitud previa y base de toda investigación es necesario(2):

- Desechar sistemáticamente todos los preconceptos, con el fin de no ocasionar sesgos, especialmente subjetivos, y de ampliar el panorama fenomenológico, ya que es una forma aplicada de la "duda metódica” de Descartes. No presuponer, sino estudiar el fenómeno en sí mismo, con mente abierta a cualquier resultado obtenido por una correcta metodología.

- Tomar como objeto de las investigaciones fenómenos definidos previamente por ciertos caracteres exteriores que les son comunes, e incluir en la misma investigación a todos los que responden a esta definición. Esta recomendación se desenvuelve en las variables y muestra representativa de un protocolo. 
- Abordar los hechos sociales desde un ángulo en que se presenten aislados de sus manifestaciones individuales. La intención es inductiva y se pretende llevar las conclusiones a cierto grado de generalización.

El protocolo(3) o diseńo de la investigación es el conjunto de decisiones: es el plan y la estructura que deben adoptarse para obtener respuestas a las preguntas del estudio.

El tema es el primer problema que enfrenta el investigador. Para poder cumplir un cronograma, es recomendable que se elija un tema de interés personal y de preferencia sobre algo en que se tenga conocimientos previos, ya que la formación del marco conceptual tendría adelanto.

Ya definido el tema, hay que adentrarse en éste a través de una investigación bibliográfica exhaustiva, hasta llegar al "estado del arte", es decir, a ser "un experto" en el tema general. Este paso lleva una gran parte del tiempo de la investigación, pues tiene que desembocar en la elaboración de un marco teórico desde el cual desarrollar el proyecto. En este momento se eligen autores base, filosofías, teorías, todo ello a través del contraste y la síntesis de las diferentes opciones que se estudiaron y que serán las fuentes de la propia investigación.

Estas fuentes tienen diferentes grados de validez. Las primarias son aquellos documentos o personajes que intervinieron en eventos originales. En el caso documental, se refieren a los escritos directos del autor, en su propio idioma. El investigador deberá tener el suficiente conocimiento del idioma del autor y contar con las facilidades para obtener su obra, caso en ocasiones de difícil solución, pues algunos textos solamente se encuentran en acervos especializados en otro Estado o país diferente al de la residencia del investigador. Otra fuente, de menor calidad, son los textos secundarios, que se refieren a traducciones del texto original. Su problema es que pueden contener sesgos muy importantes, pues en ocasiones el traductor interpreta el sentido del autor, lo cual se evita en la lectura del texto original. En este tipo de referencias habrá siempre que consignar en las fichas los nombres de los traductores, pues esto es un parámetro de confiabilidad. En tercera categoría se encuentran textos varios, con datos indirectos de los hechos, autores sin prestigio entre expertos, textos anecdóticos que, según cada tipo de estudio, pueden tener algún grado de validez.
Al conocer en forma suficiente un tema afloran los problemas y la justificación del estudio, el valor potencial de la investigación, la viabilidad y sus consecuencias: ¿para qué sirve? ¿Qué alcance social tiene? ¿Ayuda a resolver un problema práctico? ¿Llenará un hueco de conocimiento? ¿Puede sugerir ideas, recomendaciones, hipótesis a futuros estudios? ¿Ayuda a la definición de un concepto? Las respuestas a estas preguntas son importantes pues justifican la inversión de tiempo y recursos económicos que todo proyecto conlleva.

Encontrar el problema y circunscribirlo es clave en la investigación. La recomendación es escoger un tema lo más específico posible y tratarlo con la mayor profundidad. La tendencia general es "perderse" frente a todas las ideas que surgen al elaborar los marcos y querer abarcar temas muy amplios o compuestos que, por sí mismos, son asuntos de otras tesis. Hay que disponer de suficiente tiempo en el cronograma para elegirlo, pues es punto crucial del protocolo. En este momento hay que preguntarse: ¿existe viabilidad para su realización? Deben considerarse las capacidades del investigador, los tiempos, los idiomas que debe dominar, la posibilidad y necesidad de viajes, la adquisición de libros, materiales y otros.

Como ejemplo, si la persona se interesa en el tema general de "muerte cerebral", elaborará su marco teórico sobre éste, pero su problema deberá ser más preciso; por ejemplo, "muerte cerebral en prematuros", el cual también es amplio, quizás debe circunscribirse más: "problemas bioéticos de la muerte cerebral posquirúrgica en prematuros".

Es útil en este nivel considerar el problema como si fuera "el nombre y apellido" de lo que se desarrollará, pues eso evita extraviarse en la vastedad del tema general. Por ejemplo, si se eligió "problemas bioéticos de la muerte cerebral posquirúrgica en prematuros", cada vez que se enuncien problemas u objetivos ellos deberán contener estos términos. Eso permite que seleccionemos las lecturas solamente con relación a este tema, lo cual ahorrará tiempo en la investigación.

Del problema se deriva la hipótesis, si se requiere, y la pregunta, otra clave en la investigación. No toda tesis cualitativa requiere hipótesis, pues para ello han de considerarse variables, pero siempre surgen varias preguntas, precisamente al conocer con profundidad el problema. 
A veces es difícil concretar una pregunta. Me parece que, en este punto, una lista de ellas, basada en las de Bernal, sigue vigente:

\begin{tabular}{|l|l|}
\hline Objeto de estudio & ¿Qué? \\
\hline Cronología & ¿Cuándo? \\
\hline Axiomas & ¿Quién? \\
\hline Método & ¿Cómo? \\
\hline Ontología & ¿Qué es? \\
\hline Tecnología & ¿Con qué? \\
\hline Teleología & ¿Para qué? \\
\hline Topografía & ¿Dónde? \\
\hline Ecología & ¿Contra qué? \\
\hline Etiología & ¿Cuál es la causa? \\
\hline Experiencia & ¿Consecuencias, valoración, cambios, etc.? \\
\hline
\end{tabular}

El objetivo general se deriva de la pregunta y quizás la forma más sencilla es formularlo en positivo o negativo eligiendo un verbo en la frase que indique la acción que se pretende. Por ejemplo: "analizar las consecuencias del diagnóstico de muerte cerebral posquirúrgica en prematuros en la bioética". O bien: "realizar una propuesta bioética sobre quién debería constatar la muerte cerebral posquirúrgica en prematuros".

Esta serie de preguntas también pueden ser útiles para construir los objetivos secundarios, los cuales deben, en su conjunto, responder al objetivo general. Los objetivos secundarios son el desglose de todo lo que incluye el objetivo general y pueden ser la base del desarrollo de los capítulos de la tesis.

El título es la expresión del problema, la pregunta o el objetivo general, y debe representar la intención de la investigación. Se recomienda que no exceda de 12 palabras y sea conciso y preciso. Quizás extrañe considerar en este momento el título. Lo que sucede a menudo es que las personas al principio tienen una idea, pero, al profundizar el marco, el problema y la pregunta pueden encontrar una mejor propuesta. Por eso es recomendable, si la persona no tiene experiencia en investigación, hacer primero una propuesta general y sobre ella empezar a estudiar el marco para expresar un tema más concreto.

Para poder responder la(s) pregunta(s) de investigación debe elegirse una metodología. A veces existe un innecesario debate sobre si el método experimental es el único verdaderamente científico. La verdad es que el método depende de los objetivos, ninguno es mejor que otro. En estudios experimentales, las variables pueden ser modificadas y controladas, por tanto se pueden replicar fácilmente; pero las variables independientes pocas veces tienen tanta fuerza como en la realidad, pues la sociedad y el individuo son heterogéneos, con muchas más características que las consideradas en un estudio y cierto grado de indeterminación. La investigación cualitativa está cerca de las variables reales, con mayor validez externa, aunque con difícil replicación. Depende mucho de la representatividad de la muestra $y$, generalmente, no tiene control de variables.

Ninguna de los tipos de metodología es perfecta. Cada una tiene sus ventajas y desventajas.

La investigación cualitativa tiene algunos problemas intrínsecos:

- Limitación de los sentidos ante la amplitud de los fenómenos socioeducativos.

- Dificultad para captar en profundidad las conductas, aprendizajes y relaciones sociales.

- Frecuentes errores de percepción, ligados a paradigmas, estructuras mentales y cosmovisiones, inevitables en la investigación educativa.

- El observador, al formar parte de lo observado, no puede mantener una imparcialidad o neutralidad.

- Dificultad para observar un comportamiento específico en el momento de la observación.

- Generalmente, las conductas sujetas a evaluación son limitadas.

Aun así, este tipo de investigación es adecuado y valioso para un tipo de fenómenos. Por tanto, lo que se debe buscar es que cumpla con los principios básicos de una observación científica:

- Sistematización de un protocolo que incluya un marco teórico y conceptual adecuado, problema, preguntas y/o hipótesis, y objetivos.

- Ser coherente con un desarrollo que lleve a la obtención de resultados confiables y válidos.

- Consignarse documentalmente con el fin de que sea replicable.

- Que los resultados sean producto de una adecuada metodología y se sistematicen.

Si se elige la realización de un estudio cualitativo no experimental, con posterior análisis estadístico, se deberá elaborar la metodología adecuada según el estudio elegido, pero en todos se incluye como mínimo en el diseño del estudio(4) (tabla 1): 
Tabla 1

\begin{tabular}{|c|c|}
\hline Clasificación: & Original o replicativo \\
\hline $\begin{array}{l}\text { Tipo de } \\
\text { investigación: }\end{array}$ & Básica, aplicada, experimental, observacional \\
\hline $\begin{array}{l}\text { Características } \\
\text { del estudio: }\end{array}$ & Según método de observación: longitudinal o transversal; según tiempo: prospectivo o retrospectivo. \\
\hline Tipo de análisis: & Descriptivo o analítico \\
\hline Metodología: & $\begin{array}{l}\text { - Especificar el sitio en que se realizará el estudio. } \\
\text { - Universo, muestra y tamaño de la muestra: determinar la población en que se llevará a cabo el estudio y el grupo de } \\
\text { la población que se considere representativo. } \\
\text { - Definir el universo, constituido por todas las unidades de observación en el lugar y tiempo señalados, o bien la } \\
\text { fracción representativa. } \\
\text { - Especificación de criterios para el cálculo del número de sujetos. } \\
\text { - Método de selección de participantes. Señalamiento de métodos y técnicas de selección de sujetos o unidades } \\
\text { partición. Criterios de inclusión, exclusión y suspensión de los participantes: } \\
\text { Inclusión. Definición de las características o cualidades que determinan la elección de un sujeto. } \\
\text { Exclusión. Definición de las características o cualidades que impiden el acceso de un sujeto al estudio. } \\
\text { Suspensión. Definición de las características o circunstancias que ameriten la suspensión del estudio en dicho sujeto, así } \\
\text { como la suspensión del estudio en todo el grupo participante. Definir las medidas de observación que se continuarán } \\
\text { al suspender el estudio. }\end{array}$ \\
\hline $\begin{array}{l}\text { Variables del } \\
\text { estudio: }\end{array}$ & $\begin{array}{l}\text { - Especificar las características o atributos de las unidades de observación primarias y secundarias, unidades de medida } \\
\text { y las escalas de observación en que se medirán. } \\
\text { - Seleccionar la unidad primaria de observación (individuo, pareja o par interrelacionados), o secundaria (familia, } \\
\text { grupo, turno, etc.). } \\
\text { - Definir subgrupos de contraste. } \\
\text { - Describir las variables y la forma de medirlas. } \\
\text { - Definir forma de control de las variables. }\end{array}$ \\
\hline $\begin{array}{l}\text { Técnicas } \\
\text { de análisis } \\
\text { estadístico: }\end{array}$ & $\begin{array}{l}\text { - Aplicación, conforme al modelo estadístico, de las técnicas correlacionadas de análisis de datos, definición de niveles } \\
\text { y escalas de medida, selección de pruebas de hipótesis y de las regiones de aceptación o rechazo. } \\
\text { - Señalar los procedimientos para el cómputo de la información. Elaborar un esquema de los procedimientos de análisis } \\
\text { (descripción de las tendencias centrales de los datos, varianzas, diferencias y relaciones entre variables), así como las } \\
\text { técnicas de análisis. Debe existir correspondencia entre objetivos, hipótesis y técnicas de análisis. } \\
\text { - Definir técnicas, procedimientos y métodos de confiabilidad. Descripción detallada de los procedimientos que se } \\
\text { realizarán durante la investigación para comprobar validez y confiabilidad. } \\
\text { - Elaborar las hojas y formularios para la recolección de la información para cada sujeto de investigación. } \\
\text { - Para el análisis de los datos, seleccionar cuadros, tablas de recolección de datos, gráficas que se utilizarán para el } \\
\text { análisis y presentación de resultados estadísticos. }\end{array}$ \\
\hline Financiamiento: & $\begin{array}{l}\text { Aclararlo para no perjudicar el desarrollo de la investigación por falta de recursos y para que no exista un conflicto } \\
\text { de intereses. }\end{array}$ \\
\hline $\begin{array}{l}\text { Cronograma de } \\
\text { actividades: }\end{array}$ & Especificar cada actividad, entidad responsable y tiempo de ejecución. \\
\hline \multicolumn{2}{|l|}{ Aspectos éticos } \\
\hline $\begin{array}{l}\text { Clasificación de } \\
\text { la investigación: }\end{array}$ & $\begin{array}{l}\text { - Sin riesgo. } \\
\text { - Con riesgo mínimo. } \\
\text { - Con riesgo mayor al mínimo. }\end{array}$ \\
\hline $\begin{array}{l}\text { Riesgos } \\
\text { previsibles y } \\
\text { probables: }\end{array}$ & $\begin{array}{l}\text { - Establecer las posibilidades y la naturaleza de los riesgos condicionados o inherentes de la investigación. } \\
\text { - Determinar los riesgos previsibles o probables que puedan originarse en detrimento biopsicosocial de los } \\
\text { participantes. } \\
\text { - Protección frente al riesgo físico y/o emocional. Establecer los mecanismos para salvaguardar la integridad de los } \\
\text { participantes en la investigación. }\end{array}$ \\
\hline $\begin{array}{l}\text { Carta de } \\
\text { consentimiento } \\
\text { bajo } \\
\text { información } \\
\text { previa: }\end{array}$ & $\begin{array}{l}\text { - Según el tipo de riesgo, podrá hacerse desde las recomendación oficiales, pero siempre es necesario un apartado de } \\
\text { consentimiento, aun en investigaciones sin riesgo pero que impliquen contenidos éticos. } \\
\text { - Archivo confidencial de la investigación. Procedimientos para salvaguardar la privacidad de los sujetos que participan } \\
\text { en la investigación, así como para conservar sus resultados. }\end{array}$ \\
\hline
\end{tabular}


Algunos aspectos importantes en cualquier investigación:

Una buena metodología para colectar los datos(5) es esencial para resolver el problema e implica cuatro actividades:

- Definir la hipótesis y operacionalizar las variables.

- Seleccionar un instrumento de medición disponible ya validado o elaborar uno que guarde relación con las categorías e indicadores de las variables, y que comprobemos sea válido y confiable.

- Aplicar el instrumento de recolección (obtener los datos de las variables de interés).

- Codificar adecuadamente los datos.

La pretensión es obtener información. Ésta debe tener dos características esenciales: confiabilidad y validez(6). La confiabilidad es la consistencia de los resultados obtenidos; se refiere al grado en que la aplicación repetida del instrumento, al mismo sujeto u objeto, produce iguales resultados.

Además de confiable, la información debe ser válida; esto se refiere al grado en que la calificación o resultado del instrumento refleja realmente lo que estamos midiendo. No es sencillo, en ocasiones, separar en un acto varias motivaciones o dependencias; por esto, los tipos de validez están en función de evidencias como la misma validez de contenido del instrumento, que refleja los aspectos más importantes de la conducta que estamos midiendo.

También hay que precisar la evidencia relacionada con el constructo. Aquí la validez se refiere al grado en que una medición se relaciona con otras mediciones, de acuerdo con hipótesis derivadas teóricamente, y que conciernen a los conceptos. Un constructo es una variable medida que tiene lugar en una teoría o esquema teórico. Esta validez incluye tres etapas:

- Establecer y especificar la relación teórica entre variables.

- Correlacionar y analizar conceptos.

- Interpretar las evidencias.

Otra forma de validación es a través de comparación con otros instrumentos ya validados en la medición del mismo concepto. Puede también optarse por buscar la validez a través de análisis factorial.

El instrumento elegido para obtener información debe también tener validez externa.
Otras formas de obtención de estos requisitos pueden ser la técnica de triangulación de datos, comparación entre colegas o análisis de la información por otros grupos expertos.

Además de estos criterios metodológicos, no hay que olvidar que toda investigación debe cumplir también con criterios éticos, los cuales incluyen la protección de las personas en su privacidad, confidencialidad, consentimiento y todos los requisitos convenidos en las cartas de consentimiento de la información previa(7).

En una investigación tenemos dos opciones respecto del instrumento de medición: elegir uno ya desarrollado y disponible o elaborar uno nuevo, siguiendo una técnica apropiada.

Se sugiere el siguiente procedimiento para elaborar un instrumento:

- Listar las variables que se pretenden medir u observar.

- Revisar su definición conceptual y comprender su significado y las dimensiones que la integran.

- Revisar las definiciones operacionales de las variables, esto es, cómo se ha medido cada variable, ello implica precisar categorías e indicadores.

- Elegir el o los instrumentos y adaptarlos al contexto de la investigación; para este caso se deben seleccionar instrumentos cuya confiabilidad y validez se conozca.

- Elaborar o desarrollar un instrumento implica pensar en cada variable, sus dimensiones, categorías e indicadores, así como en las preguntas o ítems para cada dimensión. Cada pregunta debe estar estrechamente relacionada con las categorías de las variables.

- Indicar el nivel de medición de las variables y de cada ítem. Los niveles de medición(8) son la forma adecuada de medir cada variable(9):

Otro punto esencial es la selección y tamaño adecuado de la muestra, pretendiendo que sea lo más representativa posible. El tamaño se determina estadísticamente según el universo de estudio y existen técnicas para ello.

Elaborado el instrumento, debe aplicarse una prueba piloto para verificar su operatividad. Ésta se aplica a personas con características semejantes a las de la muestra o población. Con ello se pretende determinar si las instrucciones se comprenden y si los ítems funcionan adecuadamente. 
Después de este piloto, en el que también se realizan pruebas de confiabilidad y validez, habrá que modificar, ajustar y mejorar el instrumento, y esto debe realizarse el número de veces que sea necesario para garantizar que de él se pueda obtener información útil.

Si bien existen diferencias entre la investigación cuantitativa y la cualitativa, éstas no son de contenido sino de procedimiento y tratamiento de la información. En la investigación cualitativa es imposible eliminar la subjetividad, pero esto no es indeseado como en la cuantitativa, ya que, al no manipularse las situaciones, se obtiene información más real y la realidad tiene un componente fuertemente subjetivo e impredecible. Lo importante es estar consciente del grado de subjetividad que impregna el estudio.

\section{¿Qué tipo de investigación cualitativa?}

Puede dividirse en tres grandes campos: documental, de campo o combinada(10). Frecuentemente, el texto es la base para la reconstrucción y la interpretación. Las técnicas interpretativas tratan de describir y decodificar un fenómeno de ocurrencia natural y están más orientadas a determinar el significado del fenómeno que su cuantificación. Defienden la existencia de relaciones abiertas y permiten conocer la percepción que los sujetos tienen de su realidad, aceptando la concepción múltiple de la misma(11).

Una de las formas más frecuentes de investigación es la de tipo documental, aunque todas las investigaciones parten en su marco teórico y conceptual del mismo procedimiento. Se refiere a la investigación realizada en diferentes tipos de escritos, como libros, documentos académicos, actas, informes, revistas, biografías, diarios, archivos, entre otros. Lo más importante en este tipo de investigación es la integración adecuada de fichas, que en forma escrita (12) o digital nos sirva para obtener información precisa. También existen diversas técnicas para compilar adecuadamente esta información, de tal forma que pueda ser analizada posteriormente. En sí, esta técnica puede constituir un trabajo de investigación, como sería la producción de un artículo de revisión, catálogo o una primera parte del análisis de un autor o período específico.

La metodología documental se basa en el análisis de textos y, en el caso de la bioética, tiene varias características que pueden combinarse en la misma investigación, entre otras:
- Transdisciplinaria: ejecuta una axiomática común a un conjunto de disciplinas(13).

- Deductiva: analiza distintos contextos históricos y situacionales en material documental para referir y circunscribir.

- De concordancia: analiza factores comunes asociados a distintos hechos, respuestas sociales, discursos, leyes, etc. Parte del encuentro de elementos presentes en distintos contextos históricos con el fin de trasladarlos a las evidencias de una práctica médica actual y de su interpretación en el campo de la bioética.

- De análisis: evalúa e interpreta el fenómeno que ocurrió o está ocurriendo en un período determinado de tiempo(14,12, pp.34-58).

- Abordaje retrospectivo: recurre al reconocimiento de los sujetos y hechos históricos que dieron origen a los actuales derechos y límites de disposición del cuerpo.

- Desarrollo descriptivo: presenta situaciones, hechos, circunstancias y fenómenos para analizar problemas bioéticos desde los tres ejes de la tesis propuesta.

- Longitudinal: recolecta datos a través del tiempo en puntos o períodos específicos, para hacer inferencias respecto del cambio, sus determinantes $y$ sus consecuencias.

- No experimental: observa situaciones ya existentes, no manipulables. No requiere la aplicación de metodología cualitativa experimental.

Otra forma de investigación cualitativa es la observación de campo. Requiere contar con observación diaria para recolectar los datos e investigar los indicadores y relaciones entre variables. Para los registros pueden utilizarse diarios de campo, cámaras fotográficas, videograbadoras, etc. Lo importante es no falsear la observación y solamente constatar el fenómeno.

Una tercera forma consiste en la observación experimental o de laboratorio. El experimento es una observación directa, al estudiar mediante el empleo de los sentidos, con o sin ayuda de aparatos técnicos, hechos y fenómenos de interés social. En esta metodología el investigador considera variables que pueden ser comparables entre grupos.

Previo a la aplicación de un instrumento de recolección de datos es conveniente diseñar el formato de vaciamiento de datos y, si se elige un sistema computarizado, 
el programa adecuado. Con frecuencia, uno de los problemas de pérdida o distorsión de la información es un inadecuado formato de vaciamiento. En ocasiones, el vaciado se realiza en un formato que no puede ser utilizado para el posterior análisis computacional estadístico y esto lleva a una gran frustración y pérdida de tiempo. Otra ventaja de tener la información sistematizada adecuadamente es que se puede utilizar en investigaciones posteriores.

Si desde el diseño se ha señalado que se requerirá de una elaboración estadística, siempre deben buscarse las pruebas idóneas para cada tipo de muestra y diseño, lo cual, en ocasiones, requiere de una interconsulta a expertos. La mayoría de los alumnos consideran que su objetivo es obtener una $p$ significativa o aplicar una $c h i$ cuadrada o $t$ de Student, porque es lo más frecuente en metodología experimental, pero no siempre la muestra y variables lo permiten. Por lo tanto, es preciso determinar para qué y en qué casos se utiliza cada prueba, con el fin de obtener los mejores resultados.

Después de obtenida la información y vaciada en un instrumento de recolección y análisis, ésta deberá ser interpretada. La interpretación es la elaboración de una argumentación de hechos a través de un análisis y síntesis de los resultados, en la cual pueden caber explicaciones causales, actuales o predictivas(15).

En este paso existen varios problemas generales. En primer lugar, decidir si la información obtenida es auténtica. En la práctica, pueden suceder errores o hasta falsificación deliberada de datos por alguno de los integrantes. Muchas veces sucede que existen varias versiones de documentos que se consideran originales o que las personas encuestadas tengan un sesgo en su información. En ocasiones, el investigador debe preguntarse si la información posee suficiente credibilidad y evaluar las condiciones en que se obtuvo. Además, debe preguntarse si esa información es representativa, esto es, si no ha habido sesgo por carga "creencial" del autor, o si no se ha manipulado información verda- dera, de tal forma que sólo aparezcan resultados que comprueben la hipótesis o respondan la pregunta en la forma que el autor considera correcta. La interpretación adecuada del significado de los resultados es una tarea analítica que, en ocasiones, requiere ser triangulada con otros investigadores, con el fin de evitar sesgos.

Otro punto importante es la precisión conceptual y terminológica. La definición de términos desde el inicio del trabajo es una herramienta útil para no caer en discusiones infundadas.

Los resultados deben siempre expresar únicamente los datos llanos, mientras que las conclusiones expresan la interpretación objetiva de estos resultados. El documento debe contar con validez interna y externa, esto es, estar basado en fuentes fidedignas y reconocidas en el campo de expertos, producto de haber consultado y obtenido las fuentes necesarias para poder realizar el análisis, confrontado propuestas, buscando sus coincidencias y disparidades, y haber desarrollado una línea coherente en todo el proceso.

¿Cómo analizar si un estudio tiene características científicas en metodología cualitativa? Respondiendo a los parámetros de cualquier tipo de investigación, esto es: ¿son adecuadas sus preguntas? ¿Contiene objetivos precisos? ¿Su hipótesis es clara? ¿Se eligió adecuadamente el tipo de investigación según el tipo de variables? ¿El tipo de diseño es adecuado? ¿Es adecuado el tamaño de la muestra? ¿Presenta pruebas de validez y confiabilidad? ¿El manejo estadístico es el adecuado? ¿Sus resultados son fidedignos y claros? ¿Su interpretación es objetiva?

Por último, la mayoría de los estudios finalizan en los resultados, pero sería mejor, basados en la experiencia obtenida, poder realizar alguna propuesta, sea de continuación de la misma línea, sean sugerencias operativas o nuevas preguntas o hipótesis que permitirán proseguir en esta búsqueda de conocimiento.

\section{Referencias}

1. Muñoz Razo C. Cómo elaborar y asesorar una investigación de tesis. México: Prentice Hall. México; 1998: 14.

2. Durkheim E. Las reglas del método sociológico. Buenos Aires: La Pléyade; 1974: 54.

3. Méndez Ramírez L. El protocolo de investigación. Lineamientos para su elaboración y análisis. México: Trillas; 1996: 3346.

4. Comité de Ética de la Investigación. Manual de procedimientos. México: Escuela de Medicina, Universidad Panamericana; 2002. 
5. Padua J. Técnicas de investigación aplicadas a las ciencias sociales. México: Fondo de Cultura Económica; 2000: 63-83.

6. Hernández Sampieri R, Fernández Collado C. Metodología de la investigación. México: McGraw-Hill; 1998: 232339.

7. Lolas F, Quezada A, Rodríguez E, (eds.) Investigación en Salud. Dimensión Ética. Santiago de Chile: CIEB Universidad de Chile; 2006.

8. Pardinas F. Metodología y técnicas de investigación en ciencias sociales. México: Siglo XXI; 1996: 166-185.

9. Bioestadística básica. En: López Jiménez F, (comp.) Manual de medicina basada en la evidencia. México: El Manual Moderno; 2004: 179-210.

10. Peñarrieta I. Introducción a la investigación cualitativa. México: ETM; 2005; 53-54.

11. Anguera MT. Axiomas comunes. En Anguera MT, et al. Métodos de investigación en psicología. Madrid: Síntesis Psicología; 1995: 513.

12. Baena G. Instrumentos de investigación. México: Editores Mexicanos Unidos; 2001: 45-68.

13. Scuratti C. Interdisciplinariedad y didáctica. La Coruńa: Edara Educación; 1977.

14. Hernández R. Metodología de la investigación. México: McGraw-Hill; 1998: 191.

15. Rodríguez R, Gil J. Metodología de la investigación cualitativa. México: Aljibe; 1998: 198-217.

Recibido: 17 de marzo de 2007

Aceptado: 30 de julio de 2007 